\begin{tabular}{|c|l|}
\hline Title & Quantization of conductance minimum and index theorem \\
\hline Author(s) & Ikegaya, Satoshi; Suzuki, Shu-Ichiro; Tanaka, Y ukio; A sano, Y asuhiro \\
\hline Citation & $\begin{array}{l}\text { Physical Review B, 94(5), 054512 } \\
\text { https://doi.org/10.1103/PhysRevB.94.054512 }\end{array}$ \\
\hline Issue Date & 2016-08-19 \\
\hline Doc URL & http://hdl.handle.net/2115/63207 \\
\hline Rights & @2016 A merican Physical Society \\
\hline Type & article \\
\hline File Information & PhysRevB.94.054512.pdf \\
\hline
\end{tabular}

Instructions for use 


\title{
Quantization of conductance minimum and index theorem
}

\author{
Satoshi Ikegaya, ${ }^{1}$ Shu-Ichiro Suzuki, ${ }^{1}$ Yukio Tanaka, ${ }^{2,3}$ and Yasuhiro Asano ${ }^{1,3,4}$ \\ ${ }^{1}$ Department of Applied Physics, Hokkaido University, Sapporo 060-8628, Japan \\ ${ }^{2}$ Department of Applied Physics, Nagoya University, Nagoya 462-8602, Japan \\ ${ }^{3}$ Moscow Institute of Physics and Technology, 141700 Dolgoprudny, Russia \\ ${ }^{4}$ Center of Topological Science and Technology, Hokkaido University, Sapporo 060-8628, Japan
}

(Received 20 April 2016; published 18 August 2016)

\begin{abstract}
We discuss the minimum value of the zero-bias differential conductance $G_{\min }$ in a junction consisting of a normal metal and a nodal superconductor preserving time-reversal symmetry. Using the quasiclassical Green function method, we show that $G_{\min }$ is quantized at $\left(4 e^{2} / h\right) N_{\text {ZES }}$ in the limit of strong impurity scatterings in the normal metal at the zero temperature. The integer $N_{\mathrm{ZES}}$ represents the number of perfect transmission channels through the junction. An analysis of the chiral symmetry of the Hamiltonian indicates that $N_{\mathrm{ZES}}$ corresponds to the Atiyah-Singer index in mathematics.
\end{abstract}

DOI: 10.1103/PhysRevB.94.054512

\section{INTRODUCTION}

The quantization of an observable value in physics is closely related some of the time to an invariant in mathematics. A good example may be the quantized Hall conductivity in condensed matter physics. Although the quantization of the Hall conductivity itself occurs for physical reasons, the quantized value is proportional to a Chern invariant in a two-dimensional manifold [1]. Another example is the number of gapless states at the surface of a topologically nontrivial material characterized by a topological invariant $Z$. The integer $Z$ depends on the spatial dimensionality and the symmetry class of the Hamiltonian [2,3]. The conductance in a junction consisting of such a topologically nontrivial superconductor is quantized at $\left(2 e^{2} / h\right) Z$ with $Z=1$ for a one-dimensional class D superconductor [4-8]. A similar phenomenon has been discussed as regards superconductors in class BDI [9-12] with $Z$ being an integer number.

The Atiyah-Singer theorem relates a topological invariant to an invariant defined in terms of solutions of a differential equation. The index theorem provides the mathematical background to the quantum anomaly in particle physics. In condensed matter physics, the index theorem describes the number of gapless modes at a boundary between two chiral superfluids [13]. When a quantized physical value is described by a mathematical invariant, the quantization should be robust under various perturbations preserving the invariant. In this paper, we show a relationship between the minimum value of the conductance in a superconducting junction and the Atiyah-Singer index.

We discuss the zero-bias differential conductance $G_{\mathrm{NS}}$ in a normal-metal/superconductor (NS) junction in two dimensions, where the normal metal contains a number of random impurities and its normal resistance is $R_{\mathrm{N}}$. The superconductor is characterized by unconventional time-reversal pairing symmetries such as $p_{x^{-}}, d_{x y^{-}}$, and $f$-wave symmetry. The analytical expression of the conductance is obtained by solving the quasiclassical Usadel equation [14-16] in a normal metal. We find that $G_{\mathrm{NS}}$ decreases to the quantized value of $\left(4 e^{2} / h\right)\left|N_{\text {ZES }}\right|$ with increasing in $R_{\mathrm{N}}$. The results suggest that $\left|N_{\text {ZES }}\right|$ is the number of perfect transmission channels in a dirty NS junction. The analysis in terms of the chiral symmetry of the Hamiltonian enables us to understand the relationship between $N_{\text {ZES }}$ and the index in the Atiyah-Singer theorem. To confirm the theoretical prediction by experiments, temperature $T$ must be low so that the thermal coherence length $\xi_{T}=\sqrt{\hbar D / 2 \pi k_{B} T}$ is longer than the length of a dirty normal metal $L$, where $D$ is the diffusion constant in the normal metal. This is the only condition that we assume in this paper.

\section{CONDUCTANCE MINIMUM}

Let us consider an NS junction described by a $2 \times 2$ Bogoliubov-de Gennes (BdG) Hamiltonian,

$$
\begin{aligned}
\hat{H}_{\mathrm{BdG}}= & {\left[\begin{array}{lc}
\xi_{\boldsymbol{r}}+V(\boldsymbol{r}) & \Delta(\boldsymbol{r}) \Theta(x) \\
\Delta(\boldsymbol{r}) \Theta(x) & -\xi_{\boldsymbol{r}}-V(\boldsymbol{r})
\end{array}\right], } \\
V(\boldsymbol{r})= & V_{\mathrm{imp}}(\boldsymbol{r}) \Theta(-x) \Theta(x+L)+v_{0} \delta(x), \\
\Delta(\boldsymbol{r}) & = \begin{cases}\Delta, & s \\
-2 \Delta \partial_{x} \partial_{y} / k_{F}^{2}, & d_{x y} \\
-i \Delta \partial_{x} / k_{F} & p_{x} \\
-i \Delta \partial_{x}\left(k_{F}^{2}+2 \partial_{y}^{2}\right) / k_{F}^{3}, & f,\end{cases}
\end{aligned}
$$

where $\xi_{\boldsymbol{r}}=-\hbar^{2} \nabla^{2} /(2 m)-\mu_{F}, m$ is the mass of an electron, $\mu_{F}$ is the chemical potential, $k_{F}$ is the Fermi wave number, and $\Theta(x)$ is the step function. We introduce the random impurity potential $V_{\text {imp }}$ in the normal metal $(-L<x<0)$ as shown in Fig. 1(a) and consider the barrier potential $v_{0}$ at the NS interface. In the $y$ direction, we apply a periodic boundary condition with $W$ denoting junction width.

The quasiclassical Usadel equation in the normal metal is represented by using $\theta$ parameterization [14-16],

$$
\hbar D \frac{\partial^{2} \theta(x, \epsilon)}{\partial x^{2}}+2 i \epsilon \sin \theta(x, \epsilon)=0,
$$

where $\epsilon$ is the energy of a quasiparticle measured from the Fermi level (zero energy). The quasiclassical Green function in a normal metal is represented in terms of $\theta$ in Eq. (4). The normal Green function $g=\cos \theta$ represents the quasiparticle excitation, whereas the anomalous Green function $f=\sin \theta$ describes the pairing correlations. They are linked to each 

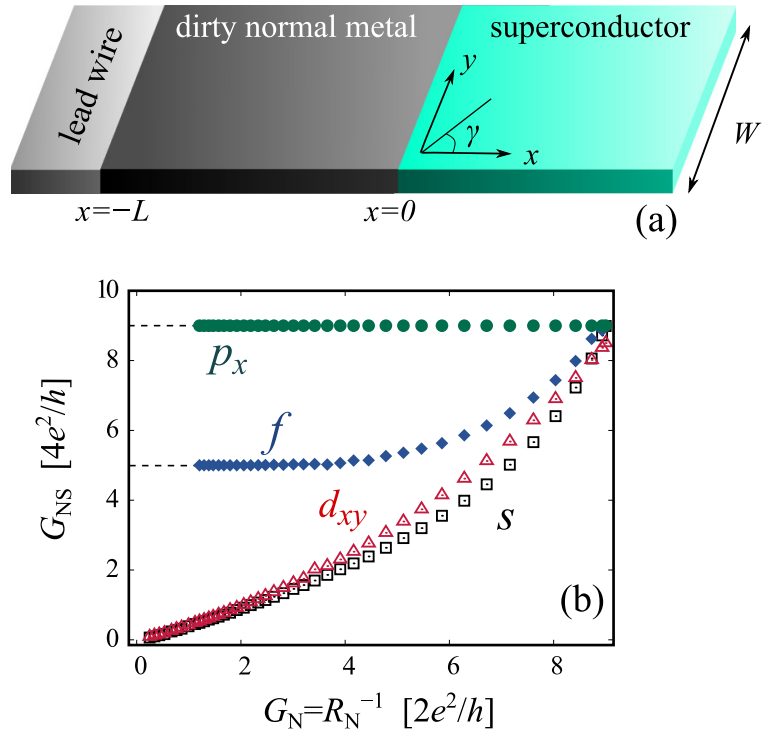

FIG. 1. (a) Schematic picture of a normal-metal/superconductor junction. (b) Numerical results on a two-dimensional tight-binding model. The index $\left|N_{\text {ZES }}\right|$ is 9 and 5 for $p_{x}$ - and $f$-wave symmetry, respectively.

other through the normalization condition $g^{2}+f^{2}=1$. The quasiclassical approximation is justified for $\Delta / \mu_{F} \ll 1$ which is satisfied in most superconductors. The Usadel equation is supplemented by two boundary conditions [17-19],

$$
\begin{gathered}
\theta(x=-L, \epsilon)=0,\left.\quad \frac{L}{G_{Q} R_{\mathrm{N}}}\left(\frac{\partial \theta}{\partial x}\right)\right|_{x=0}=2 I_{F} . \\
I_{F}=\sum_{k_{y}} F_{b c}\left(k_{y}\right) .
\end{gathered}
$$

The first boundary condition in Eq. (5) represents the absence of Cooper pairs in the lead wire because of $f=0$ at $\theta=0$. The influence of a superconductor on a normal metal is considered through the second boundary condition which represents the current conservation law. The wave number on the Fermi surface is described by $k_{x}=k_{F} \cos \gamma$ and $k_{y}=k_{F} \sin \gamma$, where $\gamma$ is the angle measured from the $x$ axis as shown in Fig. 1(a). In Eq. (6), $\sum_{k_{y}}$ means the summation over all the propagating channels whose number is calculated as $N_{c}=\left[W k_{F} / \pi\right]_{\mathrm{G}}$, where $[\cdots]_{\mathrm{G}}$ is the Gauss symbol giving the integer part of the argument. (See also the Appendix.) The pair potentials in Eq. (3) are represented as $\Delta \sin (2 \gamma)$, $\Delta \cos \gamma$, and $\Delta \cos \gamma\left(1-2 \sin ^{2} \gamma\right)$ for $d_{x y^{-}}, p_{x^{-}}$, and $f$-wave symmetries, respectively. The Andreev reflection at the NS interface is described by two pair potentials: $\Delta_{+}=\Delta(\gamma)$ and $\Delta_{-}=\Delta(\pi-\gamma)$. When $\Delta_{+}$is the pair potential for a quasiparticle incoming into the NS interface at $\gamma, \Delta_{-}$is the pair potential for an outgoing quasiparticle specularly reflected by the NS interface. In Eq. (6), $F_{b c}$ is defined by using the two pair potentials, and its expression is given in the Appendix. To calculate the zero-bias resistance at the zero temperature, the solution of the Usadel equation at $\epsilon=0$ is necessary and is described by $\theta(x)=\theta_{0}(1+x / L)$, where $\theta_{0}$ is determined by the second boundary condition in Eq. (5).
The normal resistance of a potential barrier at $x=0$ is calculated as $R_{\mathrm{B}}=\left(G_{Q} N_{c} T_{B}\right)^{-1}$ with $G_{Q}=2 e^{2} / h$ and the transmission probability of the barrier in the normal state $T_{B}$ in the Appendix. The normal resistance of a normal metal is $R_{\mathrm{N}}$ which is the inverse of the normal conductance $G_{\mathrm{N}}=$ $G_{Q} 2 \pi \hbar \rho_{0} D W / L$ with $\rho_{0}$ being the density of states at the Fermi level in the normal state. The total resistance of an NS junction at a bias voltage $V_{\text {bias }}$ is represented by a modified Ohm's law by using the Keldysh technique [17,19],

$$
R_{\mathrm{NS}}=\frac{1}{G_{Q} I_{B}}+\frac{R_{\mathrm{N}}}{L} \int_{-L}^{0} \frac{d x}{\cosh ^{2}(\operatorname{Im}(\theta(x, \epsilon)))},
$$

by putting $\epsilon \rightarrow e V_{\text {bias }}$ on the right-hand side. The first term represents the resistance due to a potential barrier, where $I_{B}$ given in the Appendix describes how the Andreev reflection modifies the resistance at the NS interface. The second term in Eq. (7) is the resistance of a normal conductor, where the integral describes how the proximity effect modifies $R_{\mathrm{N}}$. The proximity effect is a phase coherence phenomenon. Therefore, we assume that the thermal coherence length $\xi_{T}=\sqrt{\hbar D / 2 \pi k_{B} T}$ is larger than $L$ at a finite temperature $T$. In this paper, we calculate $R_{\mathrm{NS}}$ at zero temperature in the zero-bias limit.

The unconventional pair potentials in Eq. (3) have nodes on the Fermi surface. Such nodal superconductors cannot be straightforwardly classified into the ten well known topological classes [2,3]. To characterize a nodal superconductor topologically, we consider the one-dimensional Brillouin zone by fixing $k_{y}$ in the clean limit and define the one-dimensional winding number $w_{1 \mathrm{D}}[20]$. We find that

$$
w_{1 \mathrm{D}}\left(k_{y}\right)=s_{+} \frac{1-s_{+} s_{-}}{2}, \quad s_{ \pm}=\Delta_{ \pm} /\left|\Delta_{ \pm}\right| .
$$

The winding number is $s_{+}$for a channel satisfying $s_{+} s_{-}=-1$ and is zero for a channel satisfying $s_{+} s_{-}=1$. A channel with $\left|w_{1 \mathrm{D}}\right|=1$ hosts a zero-energy state (ZES) at a surface of superconductor. The three unconventional pair potentials in Eq. (3) satisfy $s_{+} s_{-}=-1$ for all the propagating channels. Therefore, such an unconventional superconductor hosts dispersionless ZESs at its clean surface [21-25].

The effects of the flat ZESs on the conductance depend on the parity of the pair potential. With spin-singlet even-parity superconductors, we can easily find that $I_{F}$ in Eq. (6) and $\theta(x)$ are real numbers at $\epsilon=0$ as shown in the Appendix. Since $\operatorname{Im}[\theta(x)]=0$, the second term in Eq. (7) becomes $R_{\mathrm{N}}$ [26]. Therefore, the zero-bias differential conductance $G_{\mathrm{NS}}=R_{\mathrm{NS}}^{-1}$ becomes

$$
\lim _{R_{\mathrm{N}} \rightarrow \infty} G_{\mathrm{NS}} \rightarrow 0
$$

for all spin-singlet even-parity superconductors. By contrast, in the spin-triplet odd-parity superconductors, we find that $I_{F}=i N_{\mathrm{ZES}}$ is a pure imaginary integer number with

$$
N_{\mathrm{ZES}} \equiv \sum_{k_{y}} w_{1 \mathrm{D}}\left(k_{y}\right)=N_{+}-N_{-} .
$$

The integer $N_{\text {ZES }}$ is defined by the summation of onedimensional winding number for all the propagation channels. 
The integer $N_{ \pm}$corresponds to the number of ZESs characterized by $w_{1 \mathrm{D}}=s_{+}= \pm 1$. A solution from the boundary condition

$$
\theta(x)=i \beta_{0}(1+x / L), \quad \beta_{0}=2 G_{Q} N_{\mathrm{ZES}} R_{\mathrm{N}},
$$

enables us to conclude that

$$
\lim _{R_{\mathrm{N}} \rightarrow \infty} G_{\mathrm{NS}} \rightarrow \frac{4 e^{2}}{h}\left|N_{\mathrm{ZES}}\right|
$$

for spin-triplet odd-parity superconductors. The derivation is given in the Appendix. The minimum value of the zero-bias conductance is quantized at a nonzero value, which suggests that $\left|N_{\text {ZES }}\right|$ is the number of perfect transmission channels through an NS junction. This is the first conclusion of this paper.

\section{ATIYAH-SINGER INDEX}

Next we analyze the physical meaning of $N_{\text {ZES }}$ by using the chiral symmetry of the Hamiltonian. The BdG Hamiltonian in Eq. (1) satisfies

$$
\left\{\hat{H}_{\mathrm{BdG}}, \hat{\Lambda}\right\}_{+}=0, \quad \hat{\Lambda}=\left[\begin{array}{cc}
0 & i \\
-i & 0
\end{array}\right],
$$

which represents the chiral symmetry of the Hamiltonian. The eigenvalue of $\hat{\Lambda}$ is either $\lambda=1$ or $\lambda=-1$. The eigenstates of $\hat{H}_{\mathrm{BdG}}$ have a characteristic property summarized as follows [20].

(i) A zero-energy state of $\hat{H}_{\mathrm{BdG}}$ is simultaneously an eigenstate of $\hat{\Lambda}$. Namely, $\hat{\Lambda} \chi_{ \pm}= \pm \chi_{ \pm}$holds for $\chi_{ \pm}$satisfying $\hat{H}_{\mathrm{BdG}} \chi_{ \pm}=0$.

(ii) On the other hand, nonzero-energy states are described by the linear combination of two states: one belongs to $\lambda=1$ and the other belongs to $\lambda=-1$. Namely $\chi_{E \neq 0}=a_{+} \chi_{+}+$ $a_{-} \chi_{-}$. Moreover the relation $\left|a_{+}\right|=\left|a_{-}\right|$always holds [27].

In what follows, we first discuss a mathematical aspect of $N_{\text {ZES }}$ briefly and show that $\left|N_{\text {ZES }}\right|$ is the number of ZESs in a dirty normal metal while taking these properties into account. By deleting the normal segment $x<0$ in Fig. 1(a), we focus on a surface of a clean semi-infinite superconductor. The wave function of a ZES localized at the surface can be represented for each propagating channel,

$$
\phi_{k_{y}}(\boldsymbol{r})=A_{k_{y}}\left[\begin{array}{c}
i \\
s_{+}
\end{array}\right] \sin \left(k_{x} x\right) e^{-x / \xi_{\gamma}} e^{i k_{y} y} \frac{1-s_{+} s_{-}}{2},
$$

where $A_{k_{y}}$ is the normalization constant and $\xi_{\gamma}=$ $\hbar v_{F} \cos \gamma /\left|\Delta_{+}\right|$depends on the pair potential in Eq. (3) and $v_{F}=\hbar k_{F} / m$ is the Fermi velocity. As suggested by property (i), $\phi_{k_{y}}$ is the eigenstate of $\hat{\Lambda}$ belonging to its eigenvalue $\lambda=s_{+}$. Since $w_{1 \mathrm{D}}=s_{+}$and $s_{+}=\lambda$, the chiral eigenvalue $\lambda$ and the one-dimensional winding number $w_{1 \mathrm{D}}$ are identical to each other. Therefore, $N_{ \pm}$in Eq. (10) is exactly equal to the number of ZESs that belong to $\lambda= \pm 1$. In Eq. (10), $N_{\text {ZES }}$ is initially defined in terms of a topological invariant. Here $N_{\text {ZES }}$ is characterized simultaneously by an invariant in terms of the solutions of the BdG equation. Mathematically, such an integer number is called the Atiyah-Singer index.

At a clean surface, the degree of degeneracy at zero energy is $N_{c}$ for three unconventional pair potentials in Eq. (3). The translational symmetry in the $y$ direction protects such
TABLE I. The key integer numbers are summarized, where $A=$ $W k_{F} / \pi, N_{c}=[A]_{\mathrm{G}}$ and $[\cdots]_{\mathrm{G}}$ means the Gauss symbol providing the integer part of the argument.

\begin{tabular}{lccc}
\hline \hline & $N_{+}$ & $N_{-}$ & $N_{\text {ZES }}$ \\
\hline$s$ & 0 & 0 & 0 \\
$d_{x y}$ & {$[A / 2]_{\mathrm{G}}$} & {$[A / 2]_{\mathrm{G}}$} & 0 \\
$p_{x}$ & $N_{c}$ & 0 & $N_{c}$ \\
$f$ & {$[A / \sqrt{2}]_{\mathrm{G}}$} & {$[A(1-1 / \sqrt{2})]_{\mathrm{G}}$} & {$[A(\sqrt{2}-1)]_{\mathrm{G}}$} \\
\hline \hline
\end{tabular}

a high degeneracy at zero energy. When we attach a dirty normal metal to form an NS junction, however, the potential disorder would lift the degeneracy at zero energy. We first count $N_{ \pm}$at a clean surface of the three unconventional superconductors as listed in Table I. Then we discuss how the potential disorder lifts the degeneracy at zero energy. With a $p_{x}$ wave, we find $N_{+}=N_{c}=N_{\text {ZES }}$ and $N_{-}=0$ because $\lambda=1$ for all the propagating channels. Such pure chiral states cannot form nonzero-energy states according to property (ii) because ZESs belonging to $\lambda=-1$ are absent [27]. This fact explains the anomalous proximity effect [18,28-30]. With a $d_{x y}$ wave, we find $N_{\text {ZES }}=0$ because the ZESs for $k_{y}>0$ $\left(k_{y}<0\right)$ belong to $\lambda=1(\lambda=-1)$. The impurity potential completely eliminates the degeneracy at zero energy because two ZESs with $\lambda=1$ and $\lambda=-1$ couple one-by-one and form two nonzero-energy states according to property (ii). This explains the absence of the proximity effect in a $d_{x y}$-wave NS junction [17,31]. The conclusion $N_{\text {ZES }}=0$ is valid for all spin-singlet even-parity superconductors. Actually $I_{F}$ in Eq. (5) is real for all spin-singlet superconductors, whereas $N_{\text {ZES }}$ is defined by the imaginary part of $I_{F}$. Finally, with an $f$ wave, we obtain $N_{\mathrm{ZES}}=\left[\left(W k_{F} / \pi\right)(\sqrt{2}-1)\right]_{\mathrm{G}}$. The integer $\left|N_{\mathrm{ZES}}\right|$ physically means the number of ZESs in the presence of potential disorder. Mathematically, $N_{\mathrm{ZES}}$ is an invariant as far as $\hat{H}_{\mathrm{BdG}}$ preserves the chiral symmetry. This is the second conclusion of this paper.

The integer $\left|N_{\mathrm{ZES}}\right|$ indicates also the number of ZESs penetrating into a dirty normal metal. The local density of states (LDOS) given by $\rho(x, \epsilon)=\rho_{0} \operatorname{Re}[\cos \theta(x, \epsilon)]$ justifies this statement. For a spin-triplet NS junction, $\theta(x)$ in Eq. (11) results in

$$
\rho(x) / \rho_{0}=\cosh \left[2 G_{Q} N_{\mathrm{ZES}} R_{\mathrm{N}}(1+x / L)\right] \gg 1,
$$

at $\epsilon=0$. The LDOS shows a drastic enhancement at zero energy, which directly indicates the penetration of the ZESs into a normal metal $[18,28,29]$. The analytical results in Eq. (12) suggest that the penetrating ZESs form the resonant transmission channels. Such degenerate ZESs in a spin-triplet junction are called Majorana flat band in recent literature [32-37].

We check the validity of the above argument by employing a numerical simulation on the two-dimensional singleband tight-binding model. We choose $L=30 a_{0}, W=25 a_{0}$, $\mu_{F} / t=1.0, \Delta / t=0.01$ with $a_{0}$ and $t$ being the lattice constant and the hopping integral, respectively. This parameter choice leads to $N_{c}=9$. In the presence of the random potential, we plot the $G_{\mathrm{NS}}$ versus $G_{\mathrm{N}}=R_{\mathrm{N}}^{-1}$ in Fig. 1(b), where the two conductances are calculated independently by using 
the recursive Green function method [38,39]. As predicted in Eq. (12), $G_{\mathrm{NS}}$ is quantized at $2 G_{Q} N_{\mathrm{ZES}}$ for spin-triplet junctions. For a $p_{x}$-wave symmetry, $N_{+}=N_{c}=9$ and $N_{-}=$ 0 are obtained numerically, which results in $N_{\mathrm{ZES}}=9$. For an $f$-wave symmetry, we find $N_{\mathrm{ZES}}=5$ because $N_{+}=7$ and $N_{-}=2$. The results also show that $G_{\mathrm{NS}}$ goes to zero with a decrease in $G_{\mathrm{N}}$ for spin-singlet $s$ - and $d_{x y}$-wave cases as predicted in Eq. (9). It is well known that the self-consistently calculated pair potential is suppressed near the interface as $\Delta(x)=\Delta \tanh \left(x / \xi_{0}\right)[22,40-42]$ in $p_{x}{ }^{-}, d_{x y}$, and $f$ cases, where $\xi_{0}=\hbar v_{F} / \pi \Delta$ is the coherence length in a superconductor. We have numerically confirmed that the conductance under such suppressed pair potential shows the same limiting behaviors as those in Fig. 1(b). The results are reasonable because the suppression of the pair potential near the interface does not break the chiral symmetry. The numerical results justify our conclusions. Although we assume a two-dimensional junction in this paper, the conclusions are valid also for a realistic three-dimensional junction. The winding number in the reduced Brillouin zone in Eq. (8) and the index in Eq. (10) are well defined in the three-dimensional junction by replacing $k_{y}$ by $\boldsymbol{k}_{\|}$, where $\boldsymbol{k}_{\|}$is the wave vector in the parallel direction to the NS interface.

\section{DISCUSSION}

The nonzero conductance minimum is a character of oddparity superconducting states that have been proposed in an artificial thin film [33] and exotic materials such as (TMTSF $)_{2} X$ ( $X=\mathrm{PF}_{6}, \mathrm{ClO}_{4}$, etc.) [43-45] and $\mathrm{Na}_{x} \mathrm{CoO}_{2} \mathrm{yH}_{2} \mathrm{O}[46,47]$. Thus the contents of this paper have a strong connection to the physics of Majorana fermion [32]. Actually, the anomalous conductance observed in experiments [6-8] in a nanowire superconductor $[4,5]$ is an example of the present theory. The Majorana fermions in a spin-triplet superconducting junction always accompany odd-frequency Cooper pairs [48-50]. The conductance quantization discussed in this paper is a part of the physics of odd-frequency Cooper pairs [29,51-53].

The topological classification of superconductors [3] cannot be applied to real superconductors straightforwardly because a number of unconventional superconductors have a nodal gap on the Fermi surface. In many cases, the nodes are necessary to realize the sign change of the pair potential on the Fermi surface which is an important factor for topologically nontrivial superconducting states. The topological characterization of such nodal superconductors is still possible in terms of a topological number defined in a restricted Brillouin zone. The dimensional reduction in the presence of the translational symmetry is the simplest method. Mirror reflection, inversion, and point group symmetries also enable us to classify symmetry-protected topologically nontrivial states [54]. A topologically nontrivial superconductor in this context has the Andreev bound states at its clean surface. According to the bulk-boundary correspondence, the degree of the degeneracy of surface bound states at zero energy should be identical to the summation of topological numbers over all the restricted Brillouin zones. Such degenerate ZESs at a surface or a junction interface cause the anomalous low energy transport which can be observed in experiments as a sign of topologically nontrivial superconducting phase. In a real superconductor, however, the potential disorder near the surface is inevitable and it may lift the degeneracy of the ZESs. This is because the potential disorder breaks the symmetries that protect the topologically nontrivial superconducting phase. In this situation, a method to measure the robustness of the degenerate ZESs is desired. In this paper, we show that the Atiyah-Singer index represents the degree of the degeneracy in the presence of potential disorder. Our method can be applied to all time-reversal superconductors because the chiral symmetry is constructed by the combination of particle-hole symmetry and time-reversal symmetry.

\section{CONCLUSION}

In summary, we have discussed the zero-bias differential conductance $G_{\mathrm{NS}}$ in a normal-metal/superconductor junction consisting of a nodal superconductor preserving time-reversal symmetry. The minimum value of $G_{\mathrm{NS}}$ is quantized at $\left(4 e^{2} / h\right) N_{\text {ZES }}$. The analysis in terms of the chiral symmetry of the Hamiltonian indicates that the integer $N_{\mathrm{ZES}}$ is the Atiyah-Singer index in mathematics.

\section{ACKNOWLEDGMENTS}

The authors are grateful to M. Sato, Ya. V. Fominov, and A. A. Golubov for useful discussions. This work was supported by "Topological Materials Science" (Nos. JP15H05852 and JP15H05853) and KAKENHI (Nos. JP26287069 and JP15H03525) from the Ministry of Education, Culture, Sports, Science and Technology (MEXT) of Japan and by the Ministry of Education and Science of the Russian Federation (Grant No. 14Y.26.31.0007). S.I. and S.S. are supported in part by a Grant-in-Aid for JSPS Fellows (Grants No. JP16J00956 and No. JP15J00797) by the Japan Society for the Promotion of Science (JSPS).

\section{APPENDIX A: BOUNDARY CONDITION}

To calculate the right-hand side of the second boundary condition in Eq. (5) in the text, the expression for $I_{F}$ is necessary. The Andreev reflection by an unconventional superconductor is described by two pair potentials,

$$
\Delta_{+}=\Delta(\gamma), \quad \Delta_{-}=\Delta(\pi-\gamma) .
$$

The Green functions in a superconductor depend on the two pair potentials as

$$
g_{ \pm}=\frac{\epsilon}{\sqrt{\epsilon^{2}-\Delta_{ \pm}^{2}}}, \quad f_{ \pm}=\frac{i \Delta_{ \pm}}{\sqrt{\epsilon^{2}-\Delta_{ \pm}^{2}}} .
$$

By using these Green functions, we define $g_{s}, f_{s}, \bar{f}_{s}$, and $z_{s}$ by

$$
\begin{aligned}
g_{s} & =g_{+}+g_{-}, \quad z_{s}=1+g_{+} g_{-}+f_{+} f_{-}, \\
f_{s} & = \begin{cases}f_{+}+f_{-} & \text {singlet } \\
i\left(f_{+} g_{-}-f_{-} g_{+}\right) & \text {triplet, }\end{cases} \\
\bar{f}_{s} & = \begin{cases}i\left(f_{+} g_{-}-f_{-} g_{+}\right) & \text {singlet } \\
f_{+}+f_{-} & \text {triplet. }\end{cases}
\end{aligned}
$$


For small $\epsilon$, we obtain the following expression

$$
\begin{gathered}
g_{s}=-i \epsilon A_{\Delta}, \quad z_{s}=1+s_{+} s_{-}-\frac{\epsilon^{2}}{\left|\Delta_{+}\right|\left|\Delta_{-}\right|}, \\
f_{s}= \begin{cases}s_{+}+s_{-} & \text {singlet } \\
\epsilon s_{+} B_{\Delta} & \text { triplet, }\end{cases} \\
\bar{f}_{s}= \begin{cases}\epsilon s_{+} B_{\Delta} & \text { singlet } \\
s_{+}+s_{-} & \text {triplet. }\end{cases} \\
A_{\Delta}=\frac{\left|\Delta_{+}\right|+\left|\Delta_{-}\right|}{\left|\Delta_{+}\right|\left|\Delta_{-}\right|}, \quad B_{\Delta}=\frac{\left|\Delta_{+}\right|-s_{+} s_{-}\left|\Delta_{-}\right|}{\left|\Delta_{+}\right|\left|\Delta_{-}\right|} .
\end{gathered}
$$

The second boundary condition in Eq. (5) in the text for $\theta(x)=\theta_{0}(1+x / L)$ results in

$$
\frac{\theta_{0}}{G_{Q} R_{\mathrm{N}}}=2 I_{F} .
$$

The expression of $I_{F}$ in Eq. (6) in the text is given by

$$
\begin{gathered}
I_{F}=\sum_{k_{y}} F_{b c}, \\
F_{b c}=\frac{\left|t_{b}\right|^{2}\left(f_{s} \cos \theta_{0}-g_{s} \sin \theta_{0}\right)}{\Xi}, \\
\Xi=\left(2-\left|t_{b}\right|^{2}\right) z_{s}+\left|t_{b}\right|^{2}\left(g_{s} \cos \theta_{0}+f_{s} \sin \theta_{0}\right),
\end{gathered}
$$

and $F_{b c}$ depends significantly on the relative sign between the two pair potentials,

$$
s_{ \pm}=\Delta_{ \pm} /\left|\Delta_{ \pm}\right| .
$$

The summation over propagating channel is evaluated as

$$
\sum_{k_{y}} \rightarrow \frac{W}{2 \pi} \int_{-k_{F}}^{k_{F}} d k_{y}=\frac{W k_{F}}{2 \pi} \int_{-\pi / 2}^{\pi / 2} d \gamma \cos \gamma=\frac{W k_{F}}{\pi} .
$$

Thus $\left[W k_{F} / \pi\right]_{\mathrm{G}}$ is the number of propagating channels $N_{c}$. In Eq. (A12), $F_{b c}$ depends on the normal transmission coefficient of a potential barrier,

$$
t_{b}=\frac{\cos \gamma}{\cos \gamma+i z_{0}}, \quad z_{0}=v_{0} /\left(\hbar v_{F}\right)
$$

where $v_{F}=\hbar k_{F} / m$ is the Fermi velocity. The summation of $\left|t_{b}\right|^{2}$ over the propagation channels gives the inverse of the normal resistance of the potential barrier $R_{\mathrm{B}}=\left(G_{Q} N_{c} T_{B}\right)^{-1}$,

$$
\sum_{k_{y}}\left|t_{b}\right|^{2}=\frac{W k_{F}}{\pi} T_{B}, \quad T_{B}=\int_{0}^{\pi / 2} d \gamma \cos \gamma\left|t_{b}\right|^{2},
$$

where $T_{B}$ is the normal transmission probability of the potential barrier.

In a spin-singlet junction, we obtain

$$
\begin{aligned}
I_{F}= & \sum_{k_{y}}\left[\frac{s_{+}\left|t_{b}\right|^{2} \cos \theta_{0}}{2-\left|t_{b}\right|^{2}\left(1-s_{+} \sin \theta_{0}\right)} \frac{1+s_{+} s_{-}}{2}\right. \\
& \left.-\tan \theta_{0} \frac{1-s_{+} s_{-}}{2}\right],
\end{aligned}
$$

at $\epsilon=0$. In an $s$-wave case, it is possible to obtain an analytical expression of the boundary condition in Eq. (A10) for $T_{B} \ll 1$.
The relation $s_{+}=s_{-}=1$ for all the propagation channels leads to

$$
\frac{R_{\mathrm{B}}}{2 R_{\mathrm{N}}} \theta_{0}=\cos \theta_{0}
$$

In the limit of $R_{\mathrm{N}} \rightarrow \infty$, we find $\theta_{0} \approx \pi / 2$. In a $d_{x y}$-wave junction, the boundary condition in Eq. (A10) becomes

$$
\frac{1}{G_{Q} R_{\mathrm{N}}} \theta_{0}=-2 N_{c} \tan \theta_{0}
$$

at $\epsilon=0$. The solution of this equation is $\theta_{0}=0$.

In a spin-singlet junction, the boundary condition in Eq. (A10) with Eq. (A18) always gives a real solution of $\theta_{0}$ at $\epsilon=0$. The proximity effect modifies the normal resistance as shown in the second term in Eq. (7) in the text. Since $\operatorname{Im}(\theta(x))=0$, the second term in Eq. (7) is always equal to $R_{\mathrm{N}}$ at zero bias.

In a spin-triplet junction, we find

$$
I_{F}=i \sum_{k_{y}} s_{+} \frac{1-s_{+} s_{-}}{2}=i \sum_{k_{y}} w_{1 \mathrm{D}}\left(k_{y}\right)
$$

at $\epsilon=0$. From the boundary condition in Eq. (A10), we obtain

$$
\theta_{0}=i \beta_{0}, \quad \beta_{0}=2 G_{Q} N_{\mathrm{ZES}} R_{\mathrm{N}} .
$$

\section{APPENDIX B: RESISTANCE IN A SPIN-TRIPLET NS JUNCTION}

The expression of the resistance in an NS junction in Eq. (7) requires a coefficient $I_{B}$ which is defined by

$$
\begin{gathered}
I_{B}=\sum_{k_{y}} \frac{\left|t_{b}\right|^{2} B}{|\Xi|^{2}} \\
B=\left|t_{b}\right|^{2}\left(1+\left|\cos \theta_{0}\right|^{2}+\left|\sin \theta_{0}\right|^{2}\right) \\
\times\left(\left|z_{s}\right|^{2}+\left|g_{s}\right|^{2}+\left|f_{s}\right|^{2}+\left|\bar{f}_{s}\right|^{2}\right) / 2+2\left(2-\left|t_{b}\right|^{2}\right) \\
\times\left[\operatorname{Re}\left(g_{s} z_{s}^{*}\right) \operatorname{Re}\left(\cos \theta_{0}\right)+\operatorname{Re}\left(f_{s} z_{s}^{*}\right) \operatorname{Re}\left(\sin \theta_{0}\right)\right] \\
+2\left|t_{b}\right|^{2} \operatorname{Im}\left(\cos \theta_{0} \sin ^{*} \theta_{0}\right) \operatorname{Im}\left(f_{s} g_{s}^{*}\right) .
\end{gathered}
$$

In a spin-triplet junction, we find

$$
\begin{aligned}
I_{B}= & 2 \sum_{k_{y}}\left[\frac{\left|t_{b}\right|^{4} \cosh ^{2} \beta_{0}}{\left(2-\left|t_{b}\right|^{2}\right)^{2}} \frac{1+s_{+} s_{-}}{2}\right. \\
& \left.+\left(\cosh ^{2} \beta_{0}-s_{+} \cosh \beta_{0} \sinh \beta_{0}\right) \frac{1-s_{+} s_{-}}{2}\right], \\
= & J_{1} \cosh ^{2}\left(\beta_{0}\right)-N_{\text {ZES }} \sinh \left(2 \beta_{0}\right), \\
J_{1}= & 2 \sum_{k_{y}}\left[\frac{\left|t_{b}\right|^{4}}{\left(2-\left|t_{b}\right|^{2}\right)^{2}} \frac{1+s_{+} s_{-}}{2}+\frac{1-s_{+} s_{-}}{2}\right]
\end{aligned}
$$

at $\epsilon=0$. By using Eq. (A22) and

$$
\frac{1}{L} \int_{-L}^{0} \frac{d x}{\cosh ^{2}\left[\beta_{0}(1+x / L)\right]}=\frac{\tanh \beta_{0}}{\beta_{0}},
$$

the resistance in Eq. (7) in the text results in

$$
R_{\mathrm{NS}}=\frac{1}{G_{Q} I_{B}}+\frac{1}{2 G_{Q} N_{\mathrm{ZES}}} \tanh \beta_{0} .
$$


When we consider the limit of $R_{\mathrm{N}} \rightarrow \infty$, the first term vanishes and $\tanh \beta_{0} \rightarrow \operatorname{sgn}\left(N_{\mathrm{ZES}}\right)$. Thus we conclude that

$$
\lim _{R_{\mathrm{N}} \rightarrow \infty} G_{\mathrm{NS}} \rightarrow \frac{4 e^{2}}{h}\left|N_{\mathrm{ZES}}\right|
$$

for a spin-triplet odd-parity superconductor.
In the absence of the pair potential (i.e., $\Delta=0$ ), a superconductor becomes an ideal lead wire. The relations $g_{ \pm}=1$ and $f_{ \pm}=0$ results in $\theta_{0}=0$ from the boundary condition at any $\epsilon$. As a result, we obtain $I_{B}=N_{c} T_{B}$ and $R_{\mathrm{NS}}=R_{\mathrm{B}}+R_{\mathrm{N}}$. The last equation represents the usual Ohm's law in the normal state.
[1] D. J. Thouless, M. Kohmoto, M. P. Nightingale, and M. den Nijs, Phys. Rev. Lett. 49, 405 (1982).

[2] A. Altland and M. R. Zirnbauer, Phys. Rev. B 55, 1142 (1997).

[3] A. P. Schnyder, S. Ryu, A. Furusaki, and A. W. W. Ludwig, Phys. Rev. B 78, 195125 (2008).

[4] R. M. Lutchyn, J. D. Sau, and S. Das Sarma, Phys. Rev. Lett. 105, 077001 (2010).

[5] Y. Oreg, G. Refael, and F. von Oppen, Phys. Rev. Lett. 105, 177002 (2010).

[6] V. Mourik, K. Zuo, S. M. Frolov, S. R. Plissard, E. P. A. M. Bakkers, and L. P. Kouwenhoven, Science 336, 1003 (2012).

[7] M. T. Deng, C. L. Yu, G. Y. Huang, M. Larsson, P. Caroff, and H. Q. Xu, Nano Lett. 12, 6414 (2012).

[8] A. Das, Y. Ronen, Y. Most, Y. Oreg, M. Heiblum, and H. Shtrikman, Nat. Phys. 8, 887 (2012).

[9] S. Tewari and J. D. Sau, Phys. Rev. Lett. 109, 150408 (2012).

[10] Y. Niu, S. B. Chung, C. H. Hsu, I. Mandal, S. Raghu, and S. Chakravarty, Phys. Rev. B. 85, 035110 (2012).

[11] M. Diez, J. P. Dahlhaus, M. Wimmer, and C. W. J. Beenakker, Phys. Rev. B 86, 094501 (2012).

[12] J. P. Dahlhaus, M. Gibertini, and C. W. J. Beenakker, Phys. Rev. B 86, 174520 (2012).

[13] G. E. Volovik, The Universe in a Helium Droplet (Clarendon Press, Oxford, 2003).

[14] G. Eilenberger, Z. Phys. 214, 195 (1968).

[15] A. I. Larkin and Yu. N. Ovchinnikov, Zh. Eksp. Teor. Fiz. 55, 2262 (1968) [Sov. Phys. JETP 28, 1200 (1969)].

[16] K. D. Usadel, Phys. Rev. Lett. 25, 507 (1970).

[17] Y. Tanaka, Yu. V. Nazarov, and S. Kashiwaya, Phys. Rev. Lett. 90, 167003 (2003).

[18] Y. Tanaka and S. Kashiwaya, Phys. Rev. B 70, 012507 (2004).

[19] Y. Tanaka, S. Kashiwaya, and T. Yokoyama, Phys. Rev. B 71, 094513 (2005).

[20] M. Sato, Y. Tanaka, K. Yada, and T. Yokoyama, Phys. Rev. B 83, 224511 (2011).

[21] L. J. Buchholtz and G. Zwicknagl, Phys. Rev. B 23, 5788 (1981).

[22] J. Hara and K. Nagai, Prog. Theor. Phys. 76, 1237 (1986).

[23] C. R. Hu, Phys. Rev. Lett. 72, 1526 (1994).

[24] Y. Tanaka and S. Kashiwaya, Phys. Rev. Lett. 74, 3451 (1995).

[25] Y. Asano, Y. Tanaka, and S. Kashiwaya, Phys. Rev. B 69, 134501 (2004).

[26] A. F. Volkov and H. Takayanagi, Phys. Rev. B 56, 11184 (1997).
[27] S. Ikegaya, Y. Asano, and Y. Tanaka, Phys. Rev. B 91, 174511 (2015).

[28] Y. Asano, Y. Tanaka, and S. Kashiwaya, Phys. Rev. Lett. 96, 097007 (2006).

[29] Y. Asano, Y. Tanaka, A. A. Golubov, and S. Kashiwaya, Phys. Rev. Lett. 99, 067005 (2007).

[30] S. Ikegaya and Y. Asano, J. Phys.: Condens. Matter 28, 375702 (2016).

[31] Y. Asano, Phys. Rev. B 63, 052512 (2001).

[32] E. Majorana, Nuovo Cimento 14, 171 (1937).

[33] J. Alicea, Phys. Rev. B 81, 125318 (2010).

[34] Y. Tanaka, Y. Mizuno, T. Yokoyama, K. Yada, and M. Sato, Phys. Rev. Lett. 105, 097002 (2010).

[35] J. You, C. H. Oh, and V. Vedral, Phys. Rev. B 87, 054501 (2013).

[36] R. Queiroz and A. P. Schnyder, Phys. Rev. B 89, 054501 (2014).

[37] A. Chen and M. Franz, Phys. Rev. B 93, 201105(R) (2016).

[38] P. A. Lee and D. S. Fisher, Phys. Rev. Lett. 47, 882 (1981).

[39] T. Ando, Phys. Rev. B 44, 8017 (1991).

[40] N. Schopohl, arXiv:cond-mat/9804064.

[41] A. A. Golubov and M. Yu. Kupriyanov, JETP Lett. 67, 501 (1999).

[42] S.-I. Suzuki and Y. Asano, Phys. Rev. B 91, 214510 (2015).

[43] D. Jerome, A. Mazaud, M. Ribault, and K. Bechgaad, J. Phys. Lett. (France) 41, 95 (1980).

[44] K. Bechgaard, K. Carneiro, M. Olsen, F. B. Rasmussen, and C. S. Jacobsen, Phys. Rev. Lett. 46, 852 (1981).

[45] K. Kuroki, R. Arita, and H. Aoki, Phys. Rev. B 63, 094509 (2001).

[46] K. Takeda, H. Sakurai, E. Takayama-Muromachi, F. Izumi, R. A. Dilanian, and T. Sasaki, Nature (London) 422, 53 (2003).

[47] K. Kuroki, Y. Tanaka, and R. Arita, Phys. Rev. Lett. 93, 077001 (2004).

[48] V. L. Berezinskii, Zh. Eksp. Teor. Fiz. Pis. Red. 20, 628 (1974). [JETP Lett. 20, 287 (1974)].

[49] Y. Asano and Y. Tanaka, Phys. Rev B 87, 104513 (2013).

[50] Y. Tanaka and A. A. Golubov, Phys. Rev. Lett. 98, 037003 (2007).

[51] Y. Tanaka, Y. Asano, A. A. Golubov, and S. Kashiwaya, Phys. Rev. B 72, 140503(R) (2005).

[52] Y. Asano, A. A. Golubov, Ya. V. Fominov, and Y. Tanaka, Phys. Rev. Lett. 107, 087001 (2011).

[53] S.-I. Suzuki and Y. Asano, Phys. Rev. B 89, 184508 (2014).

[54] K. Shiozaki and M. Sato, Phys. Rev. B 90, 165114 (2014). 